\title{
Characterization of Hydraulic Behaviours of Coarse Rock Materials in a Large Permeameter
}

\author{
Farzad Ferdos ${ }^{1}$, James Yang ${ }^{1,2}$, Anders Wörman ${ }^{1}$ \\ ${ }^{1}$ Hydraulic Engineering, Royal Institute of Technology (KTH), Stockholm, Sweden \\ ${ }^{2}$ Principal Engineer, Vattenfall Research and Development (R\&D), Älvkarleby, Sweden \\ Email: ferdos@kth.se
}

Received August 2013

\begin{abstract}
The hydraulic behaviour of a rock material structure is a major feature for its design and safety assessment. Similar to all other physical problems, in order to enclose the governing equations systems and achieve a solution, the hydraulic characteristics of these materials need to be determined experimentally and implemented then into adopted thermo-dynamical models. This paper covers the process of the design, construction and operation of an experimental rig built for this specific purpose. Using the constructed large-scale permeameter, tests have been conducted. The non-linear hydraulic behaviour of various materials under extreme turbulent conditions, where Reynolds number reaches unprecedented values, has not been studied before. Preliminary results are presented and discussed.
\end{abstract}

Keywords: Experimental Study; Permeameter; Coarse Rock Material; Turbulent Flow; Reynolds Number

\section{Introduction}

Rock materials are used in constructing a wide range of infrastructures that have interaction with water. These structures are used to control and manipulate water flow as well as to provide water retention. The structural behaviour of these structures is directly dependent on the interactions between the granules and the flowing fluid through them. Therefore, in order to calculate any structural response, a comprehensive understanding of hydraulic behaviour is needed. Among these structures, embankment dams (rock-fill dams and downstream zone of earth-fill dams) draw a lot of attention and many safety recommendations and guidelines are continuously developed nationwide to ensure the safety of these structures. This level of attention is given to dams since they are massive infrastructures built mostly of rock fill material and the consequences of their failure could prove catastrophic both socially and economically (ICOLD, 1987).

The equation most commonly used to describe the flow of a fluid through a porous medium is Darcy's law, in which the flow is expressed as a linear relation between the head loss and the fluid velocity, proportioned by hydraulic conductivity as the media's property, where the dynamic effects of the velocity are neglected (Hansen et al., 2005). For flows through coarse rockfill materials, the wide gaps between the grains allow the flow to reach higher velocities that enhance substantial dynamic forces so that the linear viscosity-based models are not appropriate to accurately account for the physics of these turbulent flows. As such, when analysing the hydraulic behaviour of these course materials, the level of turbulence must be considered to establish the gradient fields and calculate any structural response.

In spite of the numerous publications on the subject (McCouquodale \& Hannoura, 1977), most studies cover the be- haviour under moderate through-flow conditions and are conducted on material smaller in size than the actual material used in structures. These limitations make it uncertain to use these studies results and their developed formulas to study a situation where the material is relatively coarser and the pore Reynolds number, due to a higher through flow, is a couple of orders of magnitude higher than have been studied and observed. Therefore, there is a need to investigate and conduct tests to study the behaviour of coarser material under heavier and more turbulent flow regimes.

This paper presents the process of design, construction and operation of a large-scale permeameter built in Vattenfall Research and Development (R\&D) with the aim of fulfilling such needs.

\section{Permeameter Rig}

The aim of this project was to design and construct a largescale robust permeameter to be able to explore extensive flow conditions through various coarse rock materials and capture the hydraulic behaviour in these critical circumstances.

The design process was intended to meet the following criteria:

- Carrying out permeability tests on the coarse rock-fill materials which are normally used in hydraulic structures-specifically embankment dams.

- Carrying out tests for a broad range of flow magnitudes and pressure gradients from near laminar to completely developed turbulent flow conditions to fully cover the property ranges of material behaviour.

- Carrying out extensively high-flow and pressure-gradient experiments to replicate various possible critical and failure mood flow conditions.

In order to account for and minimize the wall effects, various 
guidelines regarding the magnitude of wall effects were considered. Dudgeon's threshold with zone of higher velocity concept (1966) was used to estimate the induced errors due to the wall effect in the tests. The test unit of the permeameter column was built with a larger diameter than the test material to minimize these errors. The permeameter column was constructed to be twice the length of the diameter to minimize size-effect errors. A horizontal flow set-up was selected to keep a uniform flow condition throughout the sample. The sample was fixed in place inside the test unit of the permeameter in between two metal grids in order to preserve a steady state condition by preventing them from moving due to the induced forces. 8 metal circular bars, $10 \mathrm{~mm}$ in diameter, are welded to the interior surface of the testing unit, with a $200 \mathrm{~mm}$ gap in between. They are installed to provide roughness, replicate the continuity of the material, diminish the higher velocity zones' extension, distribute the loads over the length and prevent bulk movements.

Figure 1 and Figure 2 show schematic sketches of the apparatus with the dimensions and the structural units and Figure 3 illustrates photos taken from the rig and the test

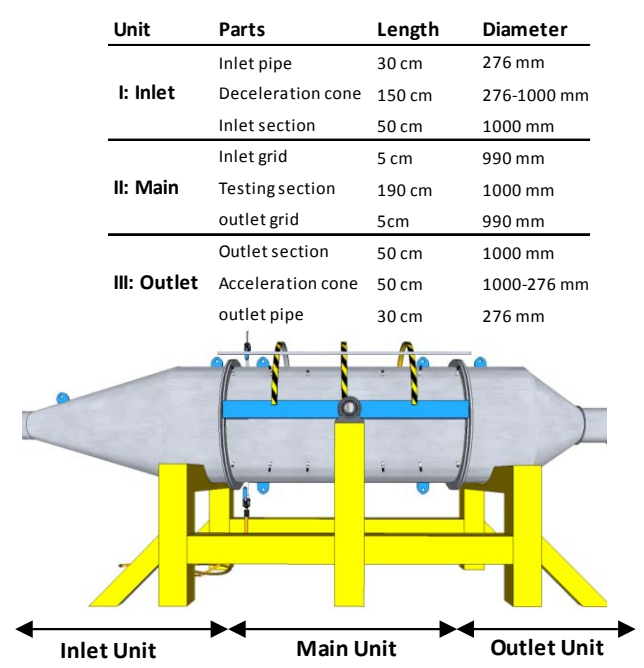

Figure 1.

Permeameter units together with the dimensions.

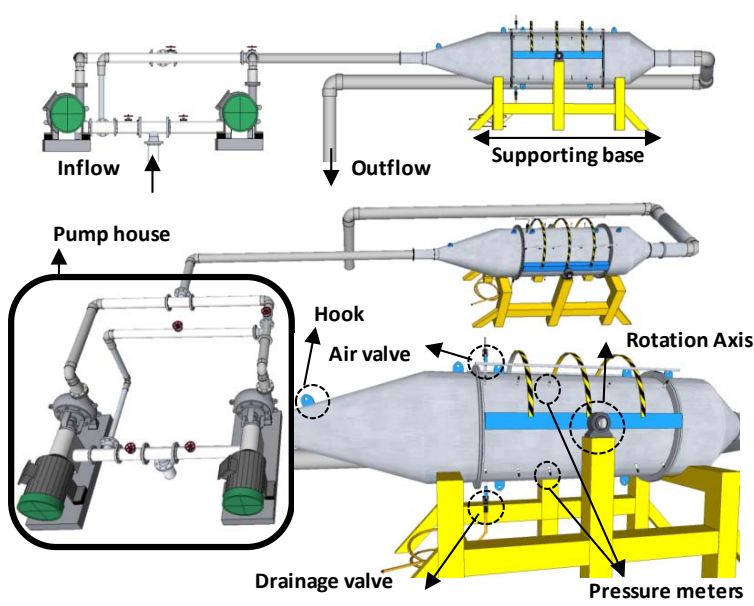

Figure 2.

Illustration of rig design and set-up.

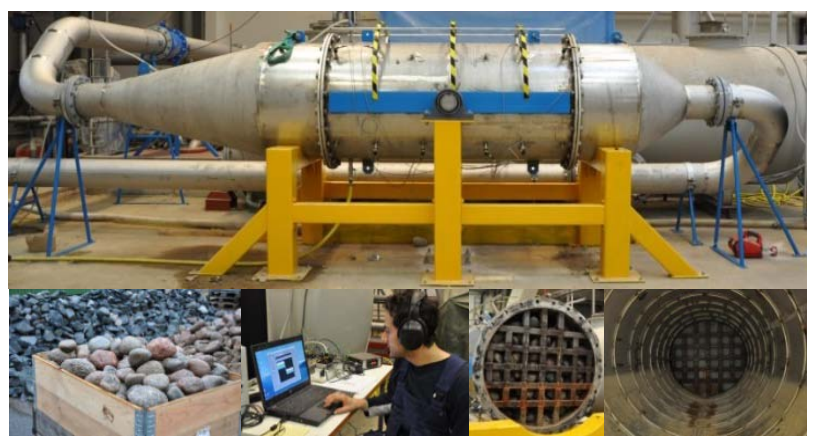

Figure 3.

Pictures of the rig, tested material and final assembling.

material during the set-up and the tests. As it is illustrated in the figures, the permeameter is constructed from stainless steel with $12.7 \mathrm{~mm}$ thickness in three sections.

Inlet unit: a funnel-shaped section with $276 \mathrm{~mm}$ - $1000 \mathrm{~mm}$ in diameter and $2000 \mathrm{~mm}$ in length which connects the inlet pipe coming from the pumping house to the main unit and disperses the flow to get a more uniform flow condition entering the sample.

Main unit: $2000 \mathrm{~mm}$ long and $1000 \mathrm{~mm}$ in diameter cylinder containing the porous media which is fixed by two $50 \mathrm{~mm}$ thick metal grids, of which one is fixed and the other is detachable.

Outlet unit: a funnel-shaped outlet unit with $1000 \mathrm{~mm}$ - 276 $\mathrm{mm}$ in diameter and $1000 \mathrm{~mm}$ length, which conduits the outcoming flow from the sample to the outlet pipeline. This takes the water back to the re-circulation basin.

The test rig was built in Vattenfall R\&D laboratory located in Älvkarleby, Sweden. This laboratory is equipped with several constant head tanks and pumping units in which, for this rig, two pumps, each with a flow capacity of $350 \mathrm{l} / \mathrm{s}$ with up to 30 $\mathrm{m}$ of water-head were used. Together these two pumps can provide $350 \mathrm{l} / \mathrm{s}$ flow with up $120 \mathrm{~m}$ of water-head (in series) or over $600 \mathrm{l} / \mathrm{s}$ with $30 \mathrm{~m}$ of water-head (in parallel). Water for the testing is supplied from one of the storage basins with sufficient storage capacity to enable continuous test conduction.

The permeameter was specifically designed to facilitate the material loading and unloading processes. The procedure for loading and unloading requires the main unit to rotate around its axis (see Figure 2) and be fixed at any angle with the help of the laboratory's overhead crane and a cable jack anchored to the floor below the apparatus. Using two motion devices together with a central axial support allows the device to be safely manipulated during both the loading and unloading sequences.

The main unit of the permeameter is attached to an anchored support structure. The inlet and outlet units are screwed to the main unit before each test run and both sit on the supporting structure. The metal supporting structure is fixed to the ground to prevent deformations and movements.

\section{Instrumentation}

The installation and selection of the monitoring instruments were planned to enable the pressure, bulk flow velocity and temperature to be measured at any time during the tests. The permeameter is equipped with 16 vibrating-wire piezometers at 6 sections along the main unit which contains the sample as illustrated in Figure 4. The sensors are installed with their 


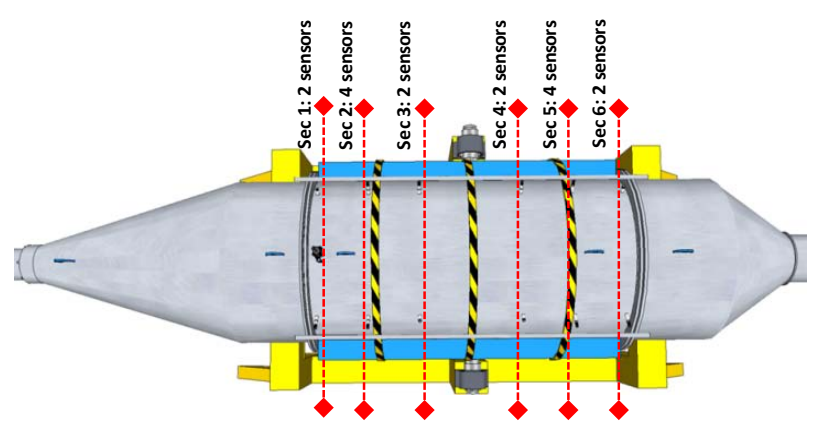

Figure 4.

Main unit with 16 piezometers which are installed diagonally in 6 sections.

heads concealed within the cylinder's wall to protect them from any damage during the loading and unloading processes. The flow through the permeameter is measured with a magnetic flow meter, installed upstream of the test rig, with are lative accuracy of less than $\pm 2 \%$. The temperature is monitored with an infrared thermometer. All the sensors were monitored and their data were recorded using a computer-controlled data acquisition system with half a second recording interval.

\section{Operation}

Each test takes approximately two days to complete, which includes preparation and loading of a sample in accordance with the safety regulations, running the pumping test and finally the unloading process. In the following section, the procedure of each test run using the permeameter is described.

\section{Loading and Unloading the Permeameter}

The washed materials in pallet rims were driven into the laboratory, using a pallet lift-truck. The inlet and outlet units of the apparatus were detached by loosening the bolts from the main unit. These units were then lifted by hooking them up to the tower crane and moving them aside. Afterwards, an explicitly designed metal plate containing a valve is screwed to the main unit. This metal plate enables the main unit to be filled with water in a vertical position and the amount of water to be measured, from which the active porosity of the sample if calculated.

For each of the tests, the permeameter was tilted at an upward angle of 20 degrees to start the filling process and gradually turned upwards to a vertical position with the help of two cranes. It was filled by carefully loading the rocks one by one into the main. Once filled, the mountable metal grid was lifted by the tower crane, placed on top of the sample and fixed with 4 screws. The main unit was then filled with water until saturation of the sample and then the volume of the water within the main unit was measured by releasing the water from the valve embedded on the metal plate. After the porosity measurement, the main unit was again tilted approximately 45 degrees backwards, towards a horizontal position, and the metal plate was then unscrewed from the main unit. Afterwards, the main unit was tilted more to reach the horizontal position, the inlet and outlet units were then lifted by the crane, mounted and screwed to the main unit. Thick rubber bands together with special filler pastes were used in the connections in order to seal them completely. The final preparation stages prior to the tests were to connect the inlet and outlet units to the inlet and outlet pipes and connect the instruments to their data-transferring and power-supplying wires.

Upon completion of each test, the wires were detached, the rig was drained using the drainage valve embedded in the bottom of the rig and then the inlet and outlet units were unscrewed. The main unit was then tilted to a vertical standing position, the metal net was unscrewed and then lifted with the help of the tower crane. The stones were then removed by hand and the unit was tilted step by step until all the stones were removed. The main unit of the permeameter was then lowered to a horizontal position, inspected and cleaned for the next test. The removed stones were then loaded onto the pallet rims once again and driven away.

The following sections describe the detailed procedure used for each test.

\section{Pre-Test Procedure}

Prior to all the tests, a procedure was performed with an empty unit while the pumps, data-acquisition system and instrumentations were checked and calibrated. After each loading and assembly of the apparatus, a $50 \mathrm{l} / \mathrm{sec}$ flow was introduced and the trapped air released with the help of the air valve embedded on top of the main unit. The connections were also inspected to ensure a proper sealing and secure connection.

\section{Testing Procedure}

After the pre-test procedure, the flow was increased in $50 \mathrm{l} / \mathrm{s}$ steps until reaching the maximum flow of $600 \mathrm{l} / \mathrm{s}$ in 12 steps. Each flow increment was maintained for approximately $5 \mathrm{~min}$. to ensure a steady state condition was reached, where all the instruments showed a constant reading with a constant fluctuation interval for that step of the flow.

\section{Material}

Two material types in two size ranges were prepared and used for the through-flow test. The material was sieved carefully to achieve a uniform size distribution (poor graded), enhancing the material grading that can represent what has been used in existing hydraulic structures or could be used for potential projects. The results for these materials can even be used to estimate the behaviour of material with slightly bigger grains and maintain the geometric property criteria outlined by Sabin and Hansen (1994).

Material characteristics are presented in Table $\mathbf{1 .}$

\section{Experimental Results}

The measurements taking during the tests were interpreted using the friction factor concept for packed columns to evaluate energy dissipation through the tested material.

In order to be able to analyse the forces exerted by the flowing fluid on the solid surfaces in a porous medium, there have been two main approaches taken in order to develop expressions for the friction factor and to assess energy losses. In the first approach, the active porosity of the porous medium is used and it is considered as a group of tangled conduits with varying cross sections, in which this theory can be further developed by using the concept of energy losses for a single straight pipe and extending it to incorporate the complicated network of pipes 
Table 1.

Properties of tested sample material.

\begin{tabular}{|c|c|c|c|c|c|}
\hline Sample & $\begin{array}{l}\text { Aggrigate } \\
\text { shape }\end{array}$ & Rock type & Density & \multicolumn{2}{|c|}{ Surface roughness } \\
\hline 1 & Cobblestone & Grenite Fedespart & 2750 & \multicolumn{2}{|c|}{$\begin{array}{l}\text { River rounded } \\
\text { smooth }\end{array}$} \\
\hline 2 & Cobblestone & Grenite Fedespart & 2750 & \multicolumn{2}{|c|}{$\begin{array}{l}\text { River rounded } \\
\text { smooth }\end{array}$} \\
\hline 3 & Crushed stone & Basalt & 2900 & \multicolumn{2}{|c|}{ Crushed rough } \\
\hline 4 & Crushed Stone & Basalt & 2900 & \multicolumn{2}{|c|}{ Crushed rough } \\
\hline Sample & $\begin{array}{l}\text { Size rang } \\
(\mathrm{mm})\end{array}$ & $\mathrm{h}^{*}$ & $\mathrm{D}_{\mathrm{p}}(\mathrm{mm})$ & $a_{v}$ & Porosity \\
\hline 1 & $100-160$ & 1.1 & 130 & 51 & 0.534 \\
\hline 2 & $160-240$ & 1.1 & 200 & 33 & 0.468 \\
\hline 3 & $100-160$ & 1.35 & 130 & 62 & 0.506 \\
\hline 4 & $160-240$ & 1.45 & 200 & 44 & 0.49 \\
\hline
\end{tabular}

${ }^{*}$ Ratio of surface area to the surface area of the equivalent sphere estimated from 50 random samples taken from each batch and dimensions compared to the available guidelines from three dimensional shape analyses.

within the medium's porosity (Bird, 2005). In the second approach, the emphasis is on the solid grains instead and the medium is considered as a group of submerged objects within a conduit. Within this framework, the energy losses and friction factor can be obtained by summing up the contribution of each of the particles to the whole energy loss (Brinkman, 1947).

For this paper, the first approach with the focus on active porosity is adopted. It is assumed that the sample has a statistically uniform packing along the length (porosity is uniform along the length) and the representative diameter of the medium is adequately small in comparison to the diameter of the permeameter.

Incorporating the assumptions allows the use of force balance in a representative tube of available tubes as:

$$
F_{k}=A K f_{\text {tube }}
$$

where $F_{k}$ is the force that is exerted by the moving fluid over the solid surfaces, $\mathrm{A}$ is the characteristic area and $\mathrm{K}$, a characteristic kinematic energy per unit volume which is proportioned by the fiction factor of the tube. Substituting the energy, area and surfaces and adopting the common mean hydraulic radius empiricism, one gets:

$$
P_{0}-P_{L}=\frac{1}{2} \rho v^{2}\left(\frac{L}{R_{h}}\right) f_{\text {tube }}
$$

In which $f_{\text {tube }}$ is the friction factor for the single representative tube, which, as can be seen from Equation (2), is a function of the Reynolds number ( $\left.\operatorname{Re}=\rho v 4 R_{h} / \mu\right) .\langle v\rangle$ is the actual velocity, $R_{h}$ is the hydraulic radius, the cross section available for the flow divided by wetted perimeter, $\rho$ is the fluid density and $\mu$ the fluid's dynamic viscosity.

From the given expression, the friction factor of a representative tube of the system can be calculated from experimental data. The friction factor for the whole column of porous media can then be analysed using the friction factor expression of a single straight pipe analogously and substituting $f_{\text {tube }}$ in to it as:

$$
\begin{aligned}
& f=\frac{1}{4}\left(\frac{D_{p}}{L}\right)\left(\frac{P_{0}-P_{L}}{\frac{1}{2} \rho v_{0}^{2}}\right) \\
& \text { where } P_{0}-P_{L}=\frac{1}{2} \rho v^{2}\left(\frac{L}{R_{h}}\right) f_{\text {tube }}
\end{aligned}
$$

In which $L$ is the length of the sample, $D_{p}$ is the effective/ mean particle diameter of the sample and $\mathrm{v}_{0}$ is the superficial velocity, which is derived by dividing the volumetric flow rate by the available void area of the cross section of the sample.

By combining the two equations we get the representative friction factor for the porous media:

$$
f=\frac{1}{4}\left(\frac{D_{p}}{R_{h}}\right)\left(\frac{v^{2}}{v_{0}^{2}}\right) f_{\text {tube }}
$$

Since the hydraulic radius can be expressed as effective porosity (n) divided by the wetted surface and at the same time bulk velocity and superficial velocity are related with the medium's effective porosity (n), the aforementioned relation can further simplified as:

$$
\begin{aligned}
R_{h} & =\frac{\text { cross section available for flow }}{\text { wetted perimeter }} \\
R_{h} & =\frac{n}{a_{v} \times(1-n)} \\
\frac{v^{2}}{v_{0}^{2}} & =\frac{1}{n^{2}}
\end{aligned}
$$

Substituting the given relations into Equation (4) results in:

$$
f=D_{p} \frac{a_{v}(1-n)}{4 n^{3}} f_{\text {tube }}
$$

In which generally, $D_{p}$ is expressed as a function of $a_{v}$ for each material as $D_{p}{ }^{p}=K / a_{v}$ where $K$ is equal to 6 for spheres and cubes and a higher value for flake shape or rod shape aggregates.

Figure 5 shows the average pressure taken from piezometer recordings at 6 sections along the main unit's length, of each flow step starting from $50 \mathrm{l} / \mathrm{s}$ until $600 \mathrm{l} / \mathrm{s}$ for each tested sample.

As can be seen from the results, a pressure profile is available for each flow step of each sample. Interpreting the results by means of the given equation for $f_{\text {tube }}$ we can evaluate the frictional material behaviour for each of the tested materials as it is illustrated in Figure 6.

From the $f_{\text {tube }}$ values in respect to the Re value, two separate behaviour zones can be observed and these are highlighted in the charts above. The initial parts show a nonlinear behaviour of $f_{\text {tube }}$ which, following some increments of flow steps and an increase of the Re, fades away. For samples 1, 3 and 4 this nonlinear behaviour is completely captured by the collected data. On the other hand, for sample 2, the test starts from a higher value for Re, and therefore only a part of this nonlinear behaviour is captured. Its missing part is marked by a hypothetical dotted black line.

From the $f_{\text {tube }}$ values with respect to the Re value and using Equation (8), the friction factor for a packed porous material column of coarse rock is calculated and presented in Figure 7. 


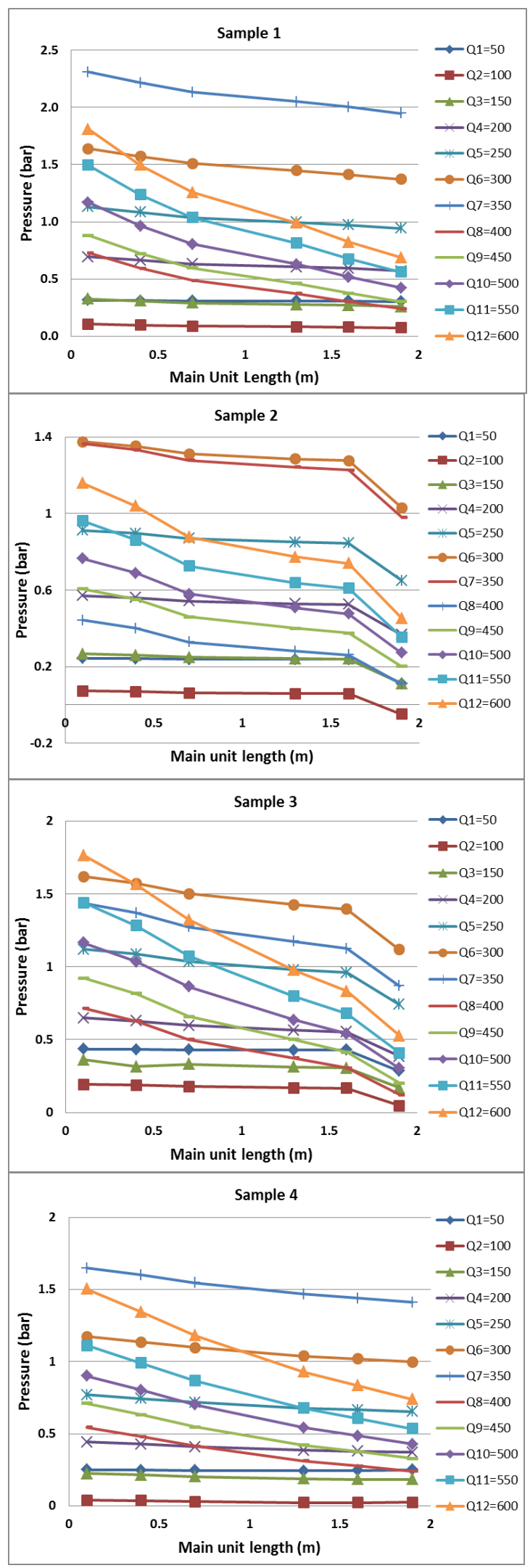

Figure 5.

Shows the averaged pressure readings from the piezometers for each flow step along the samples.

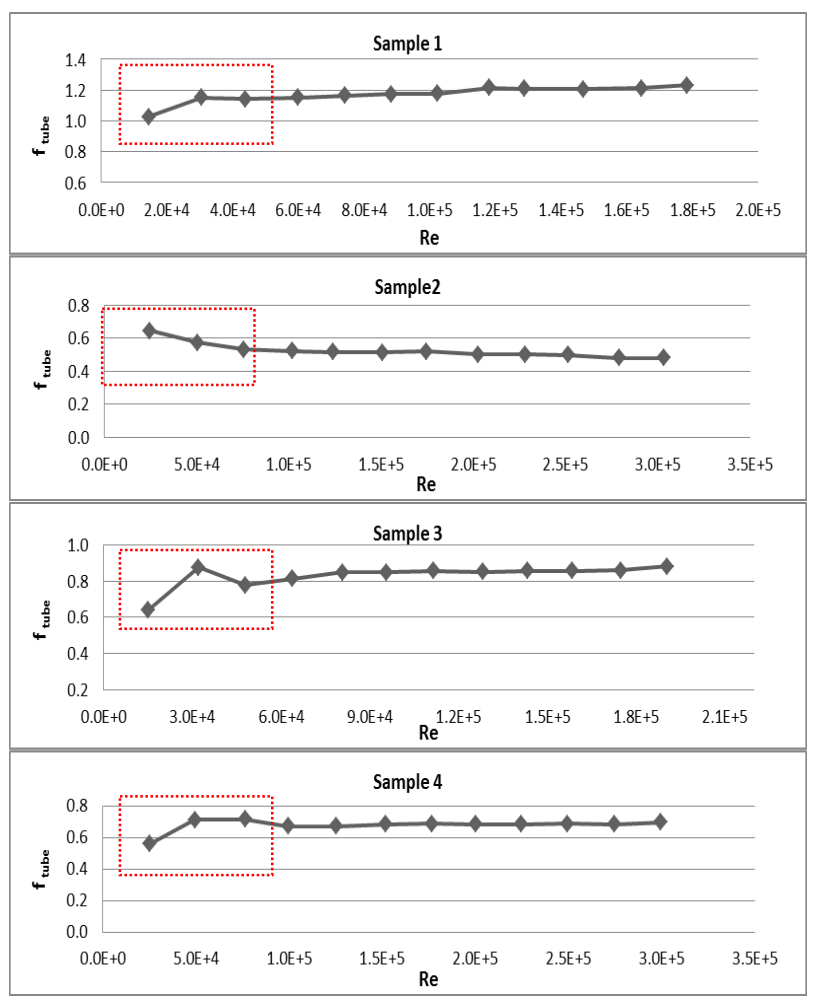

Figure 6.

Shows the $f_{\text {tube }}$ calculated for each tested materials.

\section{Conclusion}

Flow through porous structures, specifically embankment dams, is an area of growing consideration within dam structural design, safety evaluation and risk mitigation. Accidental leakage, which can be caused by internal erosion and/or differential settlements or extensive storm flows due to climate change, can result in heavy turbulent through flows with a very high Reynolds number within these structures. Forces induced by these flows can result in instabilities of slopes and even trigger dam failures. In order to be able to account for these forces, the flow regime under these critical situations needs to be investigated. For this purpose, a new experimental apparatus was constructed in Vattenfall R\&D hydraulic laboratory which facilitates wellmonitored and controlled through flow tests reaching extensive turbulence and critical gradients. A set of preliminary tests have been conducted and from the results and their interpretation it can be observed that the development of a complete, highly turbulent flow regime happens in flow conditions with a $\mathrm{Re}$ greater than 60,000 . Below this margin the friction factor is completely Re number dependent, but after the increase of flow velocity, the boundary layers around the aggregates stabilizes and the Re dependency becomes negligible. Therefore the friction factor can only be described as a function of the surface roughness without introducing significant errors on Re numbers in excess of 60,000.

\section{Acknowledgements}

This study was conducted as part of a $\mathrm{PhD}$ programme financed by the Swedish Hydropower Centre (SvensktVattenkraftcentrum, SVC), Stockholm. SVC was established by the 


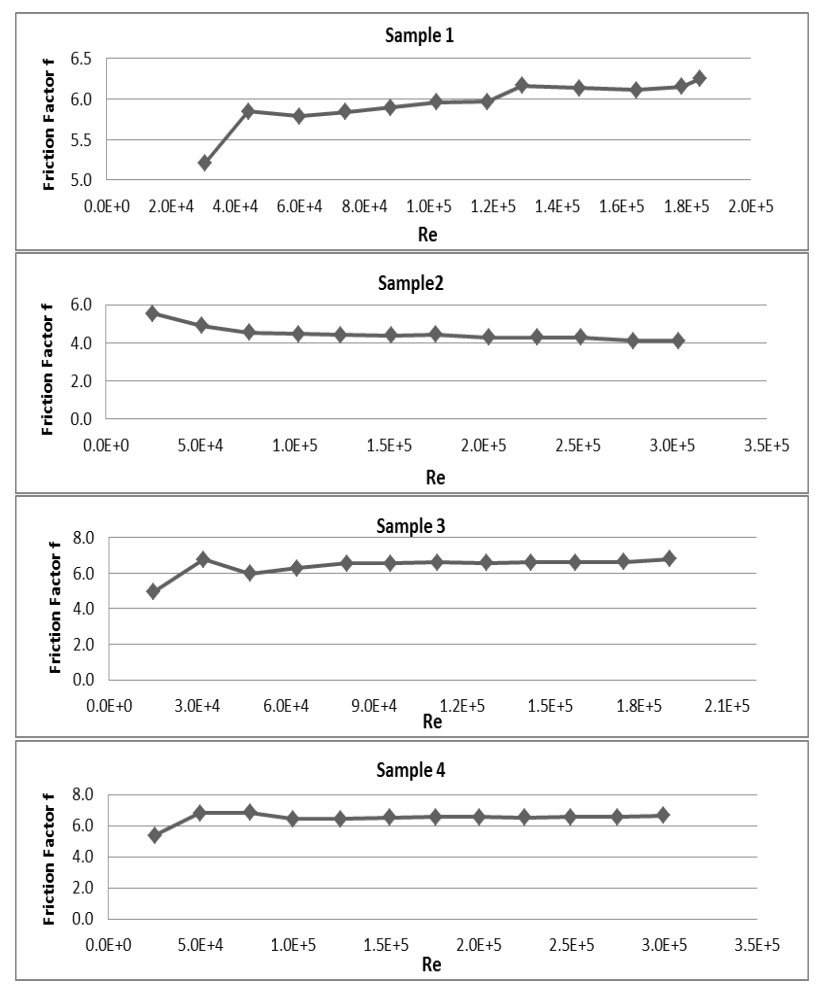

Figure 7.

Shows the friction factor calculated for each of the packed columns of rock material from the $f_{\text {tube }}$ values.

Swedish Energy Agency, Elforsk and SvenskaKraftnät, together with Luleå University of Technology, the Royal Institute of Technology, Chalmers University of Technology and Uppsala University. www.svc.nu. This experimental research is also sponsored by the Swedish Hydro-power Centre (SVC) and is being conducted in the hydraulic laboratory of Vattenfall Research and Development Centre in Sweden.

\section{REFERENCES}

Brinkman, H. C. (1947). A calculation of the viscous force exerted by a flowing fluid on a dense swarm of particles. Journal of Applied Sciences Research, A1, 27-34. URL (Last checked 20 August 2013) http://dns2.asia.edu.tw/ ysho/YSHO-English/1000\%20CE/PDF/App \%20Sci\%20Res\%20Sec\%20A1,\%2027.pdf

Byron, B. R., Stewart, W. E., \& Lightfoot, E. N. (2005). Transport phenomena (2nd ed.). New York: John Wiley \& Sons, Inc. http://dx.doi.org/10.1002/aic.690070245

Dudgeon, C. R. (1966). An experimental study of the flow of water through coarse granular media. La Houille Blanche, 7, 785-800. http://dx.doi.org/10.1051/lhb/1966049

Garboczi, E. J., Bentz, D. P., Snyder, K. A., Martys, N. S., Stutzman, P. E., Ferraris, C. F., \& Bullard, J. W. (2013). Materials and structural systems. Gaithersburg, USA: National Institute of Standards and Technology. URL (Last checked 20 August 2013) http://ciks.cbt.nist.gov/ garbocz/monograph/tablecontents.html

Hansen, D., Zhao, W. Z., \& Han, S. Y. (2005). Hydraulic performance and stability of coarse rockfill deposits, PICE (UK). Journal of Water Management, 158, 163-174. http://dx.doi.org/10.1680/wama.2005.158.4.163

ICOLD (International Commission on Large Dams) (1987). Dam safety guidelines. ICOLD Bulletin 59.

McCorquodale, J. A., Hannoura, A. A., \& Nasser, M. S. (1977). Hydraulic conductivity of rockfill. Journal of Hydraulic Research, 2, 197. http://dx.doi.org/10.1080/00221687809499625

Sabin, G. C. W., \& D. Hansen (1994). The effects of particle shape and surface roughness on the hydraulic mean radius of a porous medium consisting of quarried rock. Geotechnical Testing Journal GTJODJ, $17,43-49$ 